\title{
A Multi-perspective Narrative-Based Geovisualization Dashboard for the 2020 US Presidential Election
}

\author{
Jinmeng Rao ${ }^{1} \cdot$ Kexin Chen $^{1}$ - Ellie Fan Yang ${ }^{2}$. Jacob Kruse ${ }^{1} \cdot$ Kyler Hudson $^{1} \cdot$ Song Gao $^{1}$ (d) \\ Accepted: 13 September 2021 / Published online: 28 September 2021 \\ (C) The Author(s), under exclusive licence to Springer Nature Switzerland AG 2021
}

\begin{abstract}
In this paper, we design and implement a map dashboard that combines spatio-temporal visualization and interactive narrative to comprehensively illustrate the 2020 US presidential election. Specifically, our dashboard takes campaign rallies and major events as narrative clues and integrates multi-perspective factors (e.g., the spatial spread of COVID-19, social distancing adherence, poll results) for visualization and statistical analysis. Compared with traditional methods and products, our integrated multi-perspective solution better balances the narrative property and the geovisualization property of a dashboard, making it suitable for illustrating social or political events that happened on a large geographic scale. The result shows that our narrative-based geovisualization dashboard may be used for demonstrating and associating multiple factors with partisanship and has the potential to help users explore the interaction between policies controlling COVID-19, social distancing, and partisanship across the country during the 2020 US presidential election.
\end{abstract}

Keywords Geovisualization · Map dashboard $\cdot$ COVID-19 $\cdot$ Presidential election · Interactive narrative

This article belongs to the Topical Collection: Narrative cartography: Reconstructing place, culture, emotion and society Guest Editor: Shiliang Su

Song Gao

song.gao@wisc.edu

Jinmeng Rao

jinmeng.rao@wisc.edu

Kexin Chen

kchen264@wisc.edu

Ellie Fan Yang

fyang99@wisc.edu

Jacob Kruse

jikruse@wisc.edu

Kyler Hudson

kjhudson2@wisc.edu

1 Geospatial Data Science Lab, University of Wisconsin-Madison, 53703, Madison, WI, USA

2 School of Journalism and Mass Communication, University of Wisconsin-Madison, 53703, Madison, WI, USA

\section{Introduction}

Under the influence of the COVID-19 pandemic, the 2020 US presidential election was destined to be unusual. The spread of the coronavirus disease in the USA embodies significant spatial heterogeneity due to varying population density and structure, social economics, political attitude, and COVID-19 control policies in different places (Hou et al. 2021; Sun et al. 2020; Desmet and Wacziarg 2020), and partisan divides on state and local COVID19 response policies have become even starker (Iyengar and Westwood 2015; Sides et al. 2020). Research suggests that the COVID-19 pandemic may have caused potential political shifts (Johnson et al. 2020), and the COVID19 related health disparity issues have influenced the 2020 US election and presidential campaigns in multiple aspects (Norris and Gonzalez 2020). Meanwhile, social distancing policies such as Stay-at-Home orders issued by state and local governments have greatly affected people's daily lives, especially mobility behavior such as daily travel distance and home dwell time (Gao et al. 2020b). Recent studies also reveal that individuals' mobility and spatial interaction patterns are associated with the spread of COVID-19 and partisanship (Gao et al. 2020b; Clinton et al. 
2021; Grossman et al. 2020). Therefore, spatio-temporal visualization and analysis of COVID-19 spread, human mobility, and partisanship are of great value for exploring their interactions and the 2020 US presidential election.

Despite the growing COVID-19 confirmed cases, presidential candidates held increasingly frequent campaign rallies across the country starting in September 1, 2020 (Bernheim et al. 2020). With the help of polling data, the presidential candidates were able to easily identify battleground states (i.e., swing states, where the partisanship is not obvious) so that they could arrange more campaign rallies in these states and win their electoral votes. From a spatial perspective, the locations of the campaign rallies have significant spatial characteristics (e.g., different location preferences). From a temporal perspective, the campaign rallies also show different temporal patterns (e.g., different frequencies and trends). Such spatial and temporal characteristics make the campaign rallies excellent clues for us to track the progress of the presidential campaign across space and over time. Overall, COVID-19, mobility, partisanship, and polling provide varied perspectives for us to interpret the presidential candidates' campaign strategies and the mechanisms behind them. How to consolidate these multiple perspectives and comprehensively portray the presidential election with spatio-temporal visualization remains a large and important challenge.

For spatial phenomena, maps are one of the most common geovisualization technologies and the ability of cartographic design to meaningfully portray spatial relationships gives maps great narrative potential (Robinson 1986; MacEachren 1986; Wood and Keller 1996; Caquard and Cartwright 2014; Hanna et al. 2019). Maps with narrative elements developed in the early stage were mainly based on the compilation of static thematic maps and atlases (Wood 1987; Segel and Heer 2010; Fish 2020). One notable advantage of static maps is that users are familiar with them given their traditional use in print (Roth 2021). However, static maps are considered to have relatively limited content capacity and expression methods, and they do not work well for small-screen mobile devices (Ricker and Roth 2018). Dynamic maps greatly improve information capacity, and provide strong interactivity and rich representation methods to the map storytelling (Caquard et al. 2009; Kerski 2015; Ye et al. 2021). However, when visualizing and narrating social events and emergencies, such methods may face some challenges such as long production cycles and delayed data updates. In particular, when we need to comprehensively visualize and narrate an event from multiple perspectives throughout a specific timeline, these methods often require producing a series of static or dynamic thematic maps, making it quite challenging to preserve the consistency of style design and continuity of narrative geovisualization. The map dashboard is an emerging geovisualization technology. It has been widely used in various applications such as epidemic status tracking (Dong et al. 2020), mobility mapping (Gao et al. 2020a), and natural disaster monitoring (Tilley and Pettit 2020). A dashboard is usually described as a visual display of the most important information needed to achieve one or more objectives, consolidated and arranged on a single screen so the information can be monitored at a glance (Few 2006). With the explosive growth of geo-referenced information, the map dashboard with its dedicated components of maps and visualizations has become a popular tool that support the visual learning and analytical reasoning of geospatial knowledge (Kitchin et al. 2015; Zuo et al. 2020). Generally, map dashboards enable users to convey information by presenting location-based analytics with intuitive and interactive data visualizations on a single screen (Esri 2021). As an interactive tool, map dashboards should follow certain human-computer interaction design strategies and research methods that could facilitate users and provide user-centered geovisualization and interaction (Dransch D 2001; Hecht et al. 2011; Roth 2012; Lazar et al. 2017; Card et al. 2018). A well-designed map dashboard assures consistency in style design, which means the style and layout of a map dashboard will not be affected by the changes in time and content, thereby facilitating the data comparison and analysis across space and over time. However, map dashboards mainly focus on real-time data display and update, while lacking narrative elements. Besides, multi-perspective geovisualization (i.e., visualizing the data from multiple factors) requires a reasonable design of dashboard layout, map elements, user interface, functionality, and interactivity, which can be a highly challenging process.

To this end, we design and implement a map dashboard with a proper user-centered design that combines both spatio-temporal visualization and interactive narrative to comprehensively illustrate the 2020 US presidential election. Our work aims to address the abovementioned two challenges: (1) how to consolidate data from multiple perspectives to comprehensively portray the 2020 US presidential election and (2) how to utilize the power of map dashboards to provide proper spatio-temporal visualization and interactive narrative for demonstrating such data and portray. Specifically, we systematically sort out the campaign rallies and relevant major events from September 1 to November 3, 2020 (Election Day) and take them as narrative clues. For the same period, we collect daily COVID19 cases, social distancing metrics, poll results, and the presidential election result as multi-perspective factors, calculate statistical indicators from them, and simultaneously illustrate them in the web-based dashboard through spatiotemporal visualization. We also provide plenty of basic and composite interactive functions such as rally tracking and 
location synchronization to empower the map dashboard with the interactive narrative ability. All the components in the dashboard, including the geovisualization components and the narrative components, are internally linked together, and users' interaction with one component (data inquiry, date/location selection, etc.) will cause other elements to be updated accordingly.

The novelty of our developed dashboard is fourfold: (1) efficacy: In terms of data support and functionality, compared to build-from-scratch web map applications, our dashboard is built using ArcGIS Dashboards that is based on ESRI's tech stack and has outstanding support for various geospatial data and mature map operations, which guarantees a stable user experience. In terms of maintenance, as a dashboard maintained on the free-to-use cloud server (i.e., ArcGIS Online), all the components and data can be easily and efficiently maintained and updated on the fly. All the data and components can be copied, shared, and downloaded as needed; (2) exploratory capacity: In our dashboard, we integrate data from multiple factors (e.g., COVID-19 cases, human mobility, partisanship data) and spatially and temporally aligned them (i.e., location synchronization and spatial-temporal visualization). We also visualized all the rally activities by presidential candidates on the map. Users are able to interact with the dashboard to explore the data across space and over time; (3) processing speed: Based on ArcGIS Online, our dashboard supports building spatial indices for all the geospatial data, which speeds up the data inquiry and visualization. ArcGIS Online also provides a Content Delivery Network (CDN) that can be used to cache the layers used in the dashboard, which decreases latency when delivering comprehensive content. Practically, all the normal operations have a very short response time (responded almost instantly); however, they also rely on users' network situation. For data loading and visualization, since the dashboard and all the data are stored and maintained on ArcGIS Online, the high processing speed is guaranteed by their enterprise-level cloud server; (4) speed of comprehension: All the data are visualized as maps following scientific and aesthetic map representation methods, proper color design and legends, and all the maps are spatially aligned and temporally synchronized in the dashboard, which facilitates users' comprehension.

The intended audience for our dashboard could be anyone who is curious about the data and associations between human mobility, COVID-19 cases, partisanship, and the 2020 US presidential campaign and who would like to associate and analyze them for exploring any potential links. Our work could facilitate users since data are spatially and temporally aligned and presented in one dashboard for exploration. We provided several visualization and interaction examples introducing the use of the dashboard, and we also conducted a user evaluation to examine the design and efficacy of the dashboard. The results show that the data and functions in our dashboard are sufficient, the user experience is smooth, and the interface design is suitable. Our narrative-based geovisualization dashboard may be used for demonstrating and associating multiple factors with partisanship and has the potential to help users explore the interaction between policies controlling COVID-19, social distancing, and partisanship across the country during the presidential election.

\section{Data}

In this section, we introduce the data sources and illustrate our data collection and processing procedure. To support the multi-perspective geovisualization, we collect and process the data from various data sources. The data include daily COVID-19 confirmed cases, daily social distancing metrics (e.g., median travel distance, home dwell time), daily polling feedback, and the US presidential election results. We also collect information of the rallies and events related to the presidential candidates as narrative clues. The summary of all the data sources is shown in Table 1 . The time range for the data is from September 1 to November 3, 2020 .

\section{COVID-19 cases}

The COVID-19 case data are from the data repository maintained by the Johns Hopkins University Center for Systems Science and Engineering (JHU CSSE) (Dong et al. 2020). The data repository collects US case data at both the state level and the county or city level from the corresponding local departments of health, and the data are updated on a daily basis. We download the US county-level cumulative case data from September 1 to November 3, 2020, and formulate them as a time-series data table, where each row contains the number of the cumulative COVID19 cases in one county on a specific date. We also include the Federal Information Processing Standard Publication (FIPS) code so that each county can be uniquely represented by these codes.

After creating the COVID-19 time-series data table, we associate it with the US county map so as to produce a series of daily COVID-19 cumulative case distribution maps. The case data and the counties on the map are spatially joined based on FIPS codes. After the spatial join, each county on a specific date contains the number of cumulative cases on that date. Further dividing it by the population in that county can produce the number of cumulative cases per capita on that date. In general, the latter (i.e., COVID-19 case rate) better reflects the severity of the virus spread. 
Table 1 The summary of the data sources we used in the dashboard

\begin{tabular}{|c|c|c|}
\hline Data type & Data provider & Description \\
\hline COVID-19 Case Data & Johns Hopkins University & $\begin{array}{l}\text { The data repository collects US case data at both state level and } \\
\text { county/city level from the corresponding local departments of } \\
\text { health, and the data are updated on a daily basis. }\end{array}$ \\
\hline US Mobility Data & Descartes Labs & $\begin{array}{l}\text { The median of maximum travel distance statistics derived from } \\
\text { massive anonymized and/or de-identified mobile device locations. }\end{array}$ \\
\hline Social Distancing Metrics & SafeGraph & $\begin{array}{l}\text { SafeGraph Social Distancing Metrics data are built from an } \\
\text { anonymized population movement dataset representing } 45 \text { million } \\
\text { smartphone devices and are representative of the US population. }\end{array}$ \\
\hline Presidential Primary Polling Average & FiveThirtyEight & $\begin{array}{l}\text { A polling aggregation website that focuses on opinion poll analysis. } \\
\text { The } 2020 \text { daily Presidential Primary Polling Average data include } \\
\text { daily estimated support rates for the presidential candidates across } \\
\text { the nation, respectively. }\end{array}$ \\
\hline Campaign Rally Data & Chicago Tribune & $\begin{array}{l}\text { The campaign rally data are collected from the Campaign Trail } \\
\text { Tracker created by the Chicago Tribune. The tracker tracks the } \\
\text { campaign stops made by Donald Trump, Joe Biden, and their } \\
\text { running mates from September } 1 \text { to November } 3 \text {, 2020. The } \\
\text { GPS locations, dates, and plain-text descriptions of the rallies are } \\
\text { recorded. }\end{array}$ \\
\hline Presidential Election Major Events & Wikipedia & $\begin{array}{l}\text { The major events related to the presidential election are collected } \\
\text { from the "Timeline of the } 2020 \text { United States presidential election" } \\
\text { article on Wikipedia, which includes the major events that } \\
\text { happened on each date during the presidential election period. }\end{array}$ \\
\hline Presidential Election Result & $\begin{array}{l}\text { Fox News, Politico, The New } \\
\text { York Times }\end{array}$ & $\begin{array}{l}\text { The US county-level presidential election results including vote } \\
\text { counts and percentages scraped from Fox News, Politico, and the } \\
\text { New York Times. }\end{array}$ \\
\hline
\end{tabular}

\section{Social Distancing Metrics}

The social distancing metrics we use consist of two components: Percent Change in Mobility (PCM) and Home Dwell Time (HDT). PCM represents the percentage change in daily mobility from a mobility baseline in a specified region each day, and HDT represents the median time (hour) people spend staying at home in a specified region each day. Both can reflect people's daily mobility patterns and how they change over time, thereby revealing if people are practicing social distancing by reducing travel. We use the US mobility data released by Descartes Labs (Warren and Skillman 2020), the mobility statistics derived from massive anonymized mobile device locations, to measure the daily mobility changes from September 1 to November 3, 2020. According to the definition, mobility is represented by the maximum travel distance $(\mathrm{km})$ to a point from the initial point of the day (i.e., the max-distance mobility). For each region (e.g., a state or a county), a mobility baseline $m_{b}$ is determined to measure the changes in mobility, and it is defined as the median of the max-distance mobility on the weekdays between 2/17/2020 and 3/7/2020. Therefore, Change in Mobility (CM) can be defined as follow:

$C M=\frac{m}{m_{b}}$ where $m$ denotes the mobility (i.e., the median of the maxdistance mobility) of a specified region on a specified date, and $m_{b}$ denotes the mobility baseline for a specified region. Likewise, PCM can be calculated as follows:

$P C M=C M-1$

Where CM denotes the change in mobility from the baseline of a specified region on a specified date. For example, a PCM of $60 \%$ suggests that the mobility in this region on that date is $60 \%$ higher than the mobility baseline in this region and vice versa.

Apart from the PCM, we also use the Social Distancing Metrics data released by SafeGraph to calculate the HDT data to reveal how much time people spend at home as well as how it changes over time (Gao et al. 2020a). SafeGraph Social Distancing Metrics data are built from an anonymized population movement dataset representing 45 million smartphone devices and are representative of the US population. The home location (i.e., the common nighttime location in recent months with a precision of around 100 square meters) for each device owner is identified and the hours for all sampled devices staying at that location across the day are summed up. The devices are aggregated by home census block group, and then the median HDT for all observed devices is aggregated to the county level. Similar to COVID-19 case data, we also spatially join the PCM data, 
the HDT data and the county maps based on FIPS codes, respectively.

\section{Poll Results}

Poll results are an important contribution to the map dashboard because they provide an estimate of public opinion over the course of the presidential campaign. Polling also identifies which states may be the most competitive in the election. These results may be affected by the candidates' campaign activities or local COVID-19 policy. The polling data we use are from FiveThirtyEight, a polling aggregation website that focuses on opinion poll analysis. We collect FiveThirtyEight's daily presidential polling averages between September 1 and November 3, 2020. The polling estimates by FiveThirtyEight are weighted by the website's ratings of the pollsters, adjusting the pollsters' "house effects" (which are systematic tendencies for pollsters to favor either the Democratic or Republican candidate), and national-level polling changes. FiveThirtyEight provides only state-level, not county-level, presidential polling estimates, so we use those in our map dashboard.

Specifically, the polling data include daily estimates of the share of the state's population supporting each major party candidate. We also calculate the margins of support by comparing the estimated support rates between the two candidates. For example, a positive winning margin suggests that Joe Biden is currently leading Donald Trump in a particular state. We spatially join this daily polling data with the other data sources using the state FIPS codes.

\section{Rallies and Events}

Rallies and events serve as the key narrative clues in our dashboard, and these clues give us a detailed picture of how the presidential election goes across the nation as well as the different campaign strategies of two presidential candidates. We collect the campaign rally data from the Campaign Trail Tracker created by the Chicago Tribune. The tracker tracks the campaign trail stops made by Donald Trump, Joe Biden, and their running mates from September 1 to November 3, 2020. The GPS locations, dates, and plain-text descriptions of the rallies are recorded. We also collect the major events related to the presidential election (September 1 to November 3, 2020) from the "Timeline of the 2020 United States presidential election" article on Wikipedia, which includes the major events that happened on each date. We convert the rally data into an Esri Shapefile for spatialtemporal visualization. We formulate the event data as a data table where each row represents a specific date and contains the major events that happened on that date.

\section{Presidential Election Results}

The presidential election results indicate the level of electoral support for each candidate. The election result data we use are the US county-level results retrieved from Fox News, Politico, and the New York Times. The data contain the vote counts for the two candidates, the corresponding vote percentages, and the difference in the two percentages. We spatially join the presidential election result data with the other data sets based on the county FIPS codes.

\section{Methods}

In this section, we introduce the overall design of the dashboard and explain each component in the dashboard in detail. The overall framework of our dashboard is shown in Fig. 1. The design and implementation of our dashboard follow the standard ArcGIS Dashboards creation procedure. Specifically, we adopt the User-Centered Design principles for designing the dashboard. We focus on the users and their needs in each phase of the design process to make sure our dashboard can meet their needs. Here, the intended audience (users) for our dashboard could be anyone who is curious about the data of human mobility, COVID-19 cases, partisanship, and presidential campaign and who would like to associate and analyze them for exploring any potential links. Our work could facilitate users since data are spatially and temporally aligned and presented in one dashboard for coherent exploration. This dashboard consists of four layers: Data Preparation Layer, Data Management Layer, Methodology layer, and Application layer. Generally speaking, according to User-Centered Design, all these four layers should be designed according to the needs of users. For example, in the data preparation and management layer, users' interest in data will greatly affect the selection, processing, and organization of data. Similarly, users' demands on utilizing the data determine the provided functions and applications.

\section{Data Preparation Layer}

As introduced in Section "Data", we collect the COVID-19 infection cases, social distancing metrics, poll results, rallies and events, and presidential election results from multiple data sources and providers (including SafeGraph, Descartes Labs, JHU CSSE, The New York Times, Chicago Tribune, FiveThirtyEight, and Wikipedia). After data collection, we clean the data and check the data quality. We also ensure that the data are aggregated to the county level (except poll results, which are aggregated to the state level). Then, we match the data to the US county map or state map based on 
Fig. 1 The overall framework of the dashboard

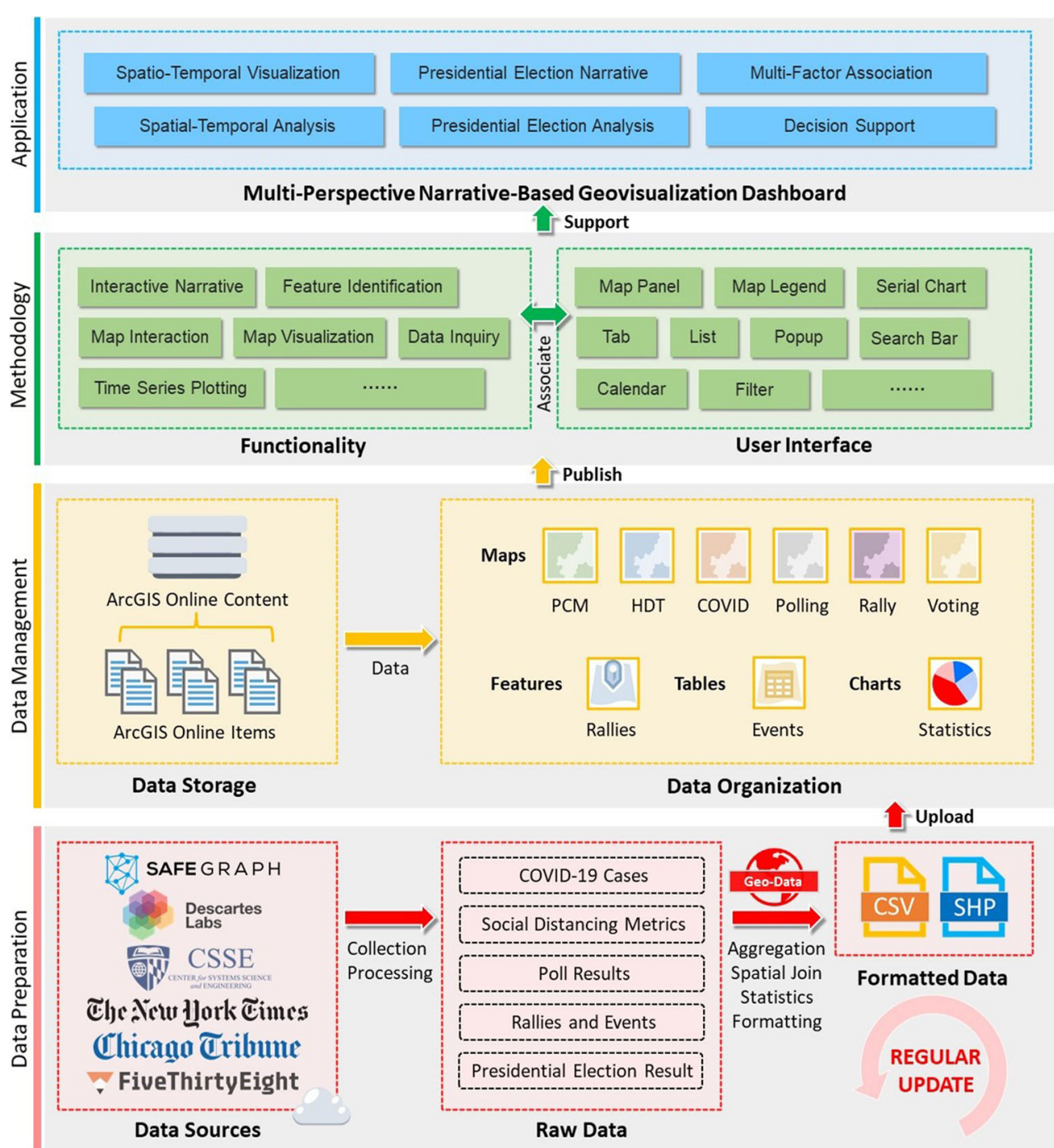

their aggregation granularity using the spatial join operator. For the rally and event data, we keep them as data tables and the GPS locations of rallies are recorded in the table for further geovisualization. Statistical indicators such as COVID-19 cases per 1000 people are calculated during this process. Lastly, all the data are formulated as Esri Shapefiles or Comma Separated Values (CSV) files and are uploaded to ArcGIS Online, a cloud-based GIS mapping platform by Esri. The whole data preparation process can be iterated regularly to keep the data correct and up to date.

\section{Data Management Layer}

In this layer, we manage all the uploaded data using ArcGIS Online. The data (both Esri Shapefiles and CSV files) uploaded from the data preparation layer are stored in ArcGIS Online Content as web items. We then further organize them as time-series tables, feature layers, and web maps that can be used for publication.
For example, COVID-19 infection cases, social distancing metrics, polling data, voting results, and rally data are hosted as feature layers and are imported into web maps for geovisualization (e.g., COVID-19 Case map, Percent Change in Mobility Map, Home Dwell Time map, Polling \& Rally Tracking Map, Rally Distribution Map, and 2020 Voting Result Map). Event data are also organized as hosted tables for inquiry.

\section{Methodology Layer}

In the Methodology Layer, we apply the functionality design and user interface design. These two parts are carefully designed and internally associated so as to improve user experience and help users better understand and interact with the data. For the functionality, we provide both basic functions and composite functions. Basic functions allow users to visit and interact with the data, and they include some fundamental operations such as 
map interaction (e.g., pan, zoom), map visualization, data visualization (e.g., charts), feature selection, data inquiry and so forth.

Composite functions are composed of several basic functions and mainly help with the interactive narrative process, which is the key feature of our dashboard. The main composite functions in the dashboard are the Rally Tracking and Location Synchronization. For rally tracking, when a user clicks on a specified rally item in the rally list, all maps will zoom into that rally location and show the all the values and geographic distributions of the corresponding COVID-19 cases, social distancing metrics, etc. There will also be a popup showing key information of that rally such as the date, city name, candidate name, and a short plain-text description of that rally. In addition, the major events happened on that date will also be shown in the narrative panel for the user's reference. For the location synchronization, we synchronize all the maps when the user changes the view of any maps. That is, when the user pans or zooms one map, all the other maps will be panned and zoomed to the specified location and zooming level. This aligns all the data well and facilitates further visual comparison and analysis.

In terms of map design and visualization, we use proper symbols and colors to represent and visualize different factors. A summary of the symbol design and color design of the maps in the dashboard is shown in Fig. 2. For the COVID-19 Cases Map, we use a choropleth map to visualize daily COVID-19 cases per 1000 people in each county, and we overlay a proportional symbol layer to further represent daily COVID-19 cases in total in each county. Moreover, for the choropleth map, we use graduated sequential colors to reflect the quantitative relationship. For the Percent Change in Mobility Map and the Home Dwell Time Map, we also use choropleth maps with graduated sequential colors to visualize the mobility index and median home dwell time in each county, respectively. For the Polling \& Rally Tracking Map, we use a choropleth map with classified diverging colors to visualize daily poll results in each state, and we overlay a point symbol map to further indicate the locations of all the rallies held by each presidential candidate over time. For the Rally Distribution Map, we visualize the locations of all the rallies as a point symbol map to reflect the rally distribution, and we use a choropleth map with graduated diverging colors to visualize the support rates of presidential candidates in the 2020 county-level voting results as a base map. For the 2020 Voting Result Map, similarly, we use a choropleth map with graduated diverging colors to visualize the 2020 county-level voting results.

ArcGIS Dashboard offers various built-in components such as map panels, chart panels (e.g., serial chart, pie chart), gauges, indicators, list, etc. Each component can visualize the data or the statistical indicators, and it can also respond to a series of preset actions (e.g., filter, component-wise association) so that the dashboard is more interactive. Thus, for the user interface design, we take advantage of these rich components and actions to support the geovisualization and interactive narrative. The overall layout design of the dashboard is shown in Fig. 3. The list panel on the left side stores the rally records, which serve as the narrative clues. The map panel groups in the middle hosts the COVID-19/social-distancing-metrics maps and presidential-election-related maps, respectively. The chart panels and indicator panels on the right side show the important statistical information and are updated based on the user's latest feature selection accordingly. The narrative panel on the bottom side tells the daily brief summary of the candidates' activities, COVID-19 cases, social distancing metrics, and the major events happened on that date.

\section{Application Layer}

This narrative-based geovisualization dashboard supports various applications such as spatio-temporal visualization and interactive narrative for the presidential election. For instance, the web maps integrated in the dashboard show the geographic variation of daily COVID-19 cases, daily social distancing metrics and the presidential election result, which may facilitate partisanship analysis (e.g., the rural-urban divide of the campaign rallies, support rate changes across space and over time), epidemic modeling (e.g., monitoring daily COVID-19 cases), and location-based analytics (e.g., spatial autocorrelation, multiple factor association analysis) on a single screen. Also, the presidential election data displayed in the dashboard and the interactive narrative function together help users track the presidential campaign and partisanship across space and over time.

\section{Results}

In this section, we demonstrate our narrative-based geovisualization dashboard, which supports spatio-temporal visualization and interactive narrative for the US 2020 presidential election. We also share some insights we found from the visualization and narrative results regarding the presidential election. The web interface of the dashboard is shown in Fig. 3 and can also be accessed at https://geods.geography. wisc.edu/covid19/election.

\section{Spatio-temporal Visualization}

The dashboard supports spatio-temporal visualization of various factors such as COVID-19 cases, social distancing metrics (including percent change in mobility and home 

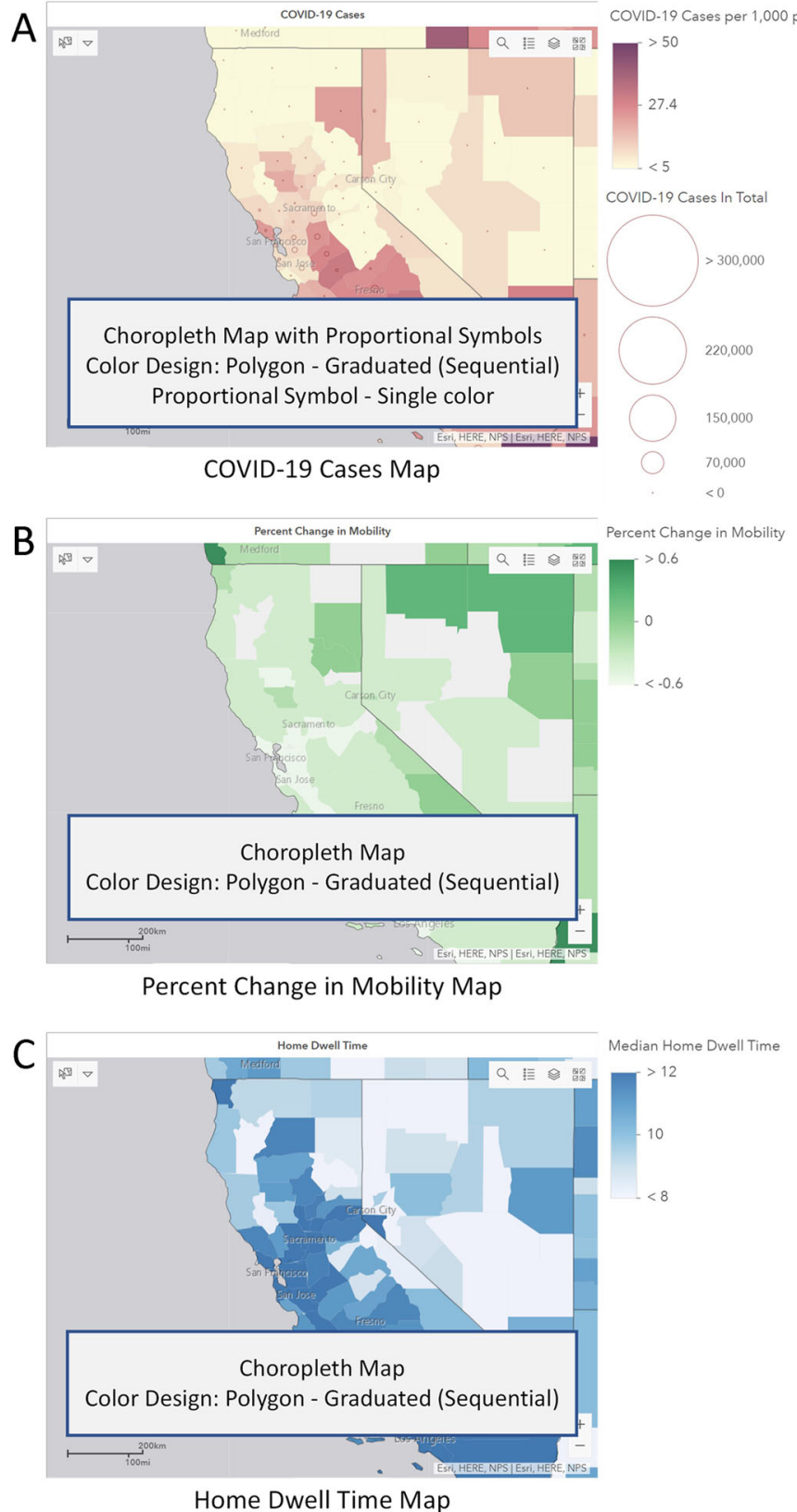
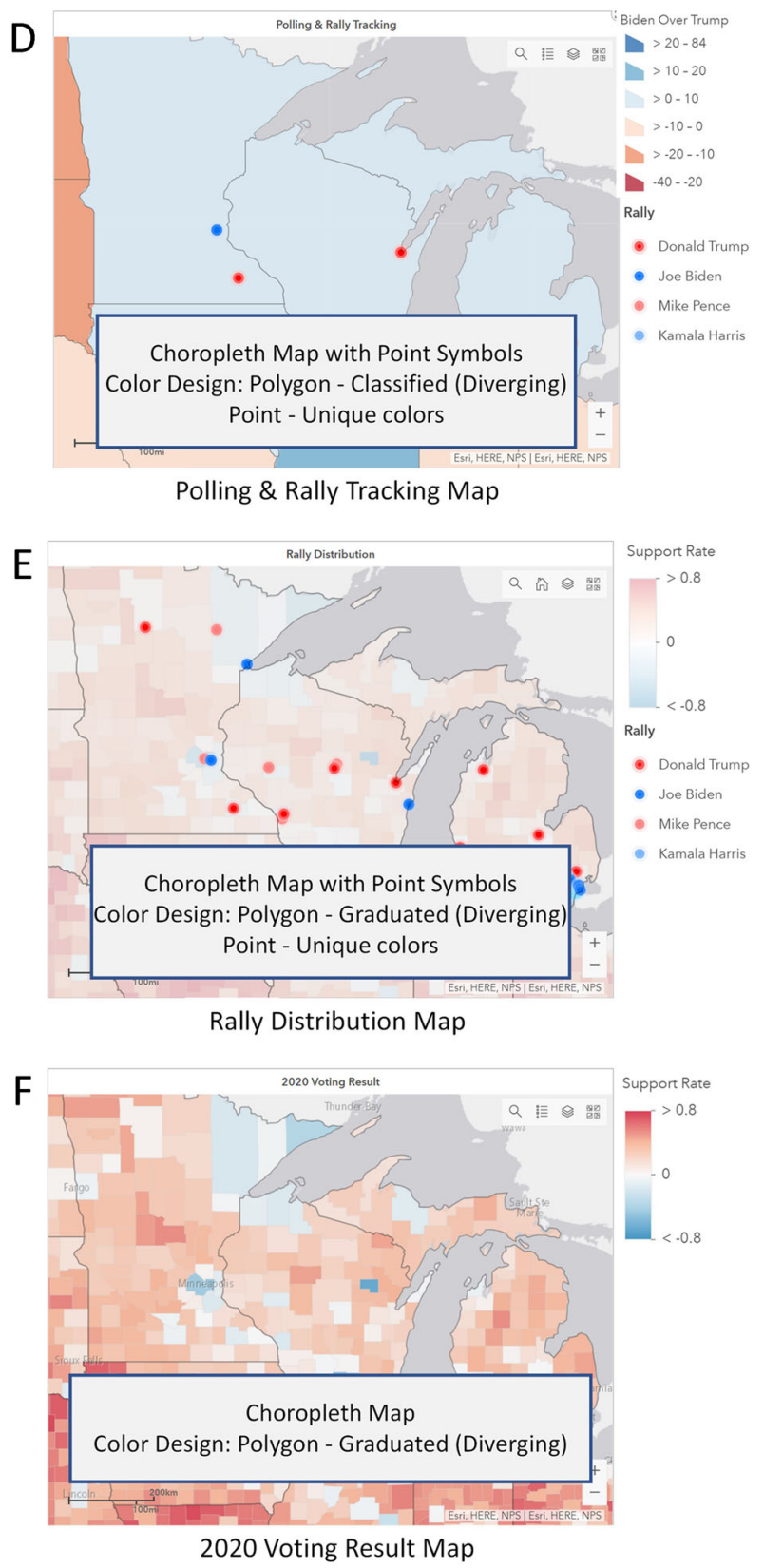

Fig. 2 The symbol design and color design of the maps in the dashboard. A COVID-19 Cases Map; B Percent Change in Mobility Map; C: Home Dwell Time Map; D Polling \& Rally Tracking Map; E Rally Distribution Map; F 2020 Voting Result Map

dwell time), rally distribution, poll results, and voting results across the country. Figure 4 illustrates several visualization examples. Please note that all the visualization examples in Figs. 4 and 5 come from the dashboard, while we rearrange the layout to avoid incomplete display (e.g., Alaska) or overlapping (e.g., toggleable legends). We show the visualization results of COVID-19 cases per 1000 people at the county level, percent change in mobility at the county level, median home dwell time at the county level, and poll results at the state level on September 1, 2020, October 6, 2020, and November 3, 2020 (the first Tuesday of each month), respectively. We can observe how these factors vary geographically across the country and how they change over time. Such spatio-temporal visualization helps users better track and understand the dynamics of the pandemic, social distancing performance, and political support. The countylevel presidential election results and the rally data can also be visualized in the dashboard. Note that we didn't show the voting results in Alaska because, according to our data source, the voting results in Alaska are collected at the election-district level while our voting result map is at the county level. Figure 5 exhibits the spatial heterogeneity of 


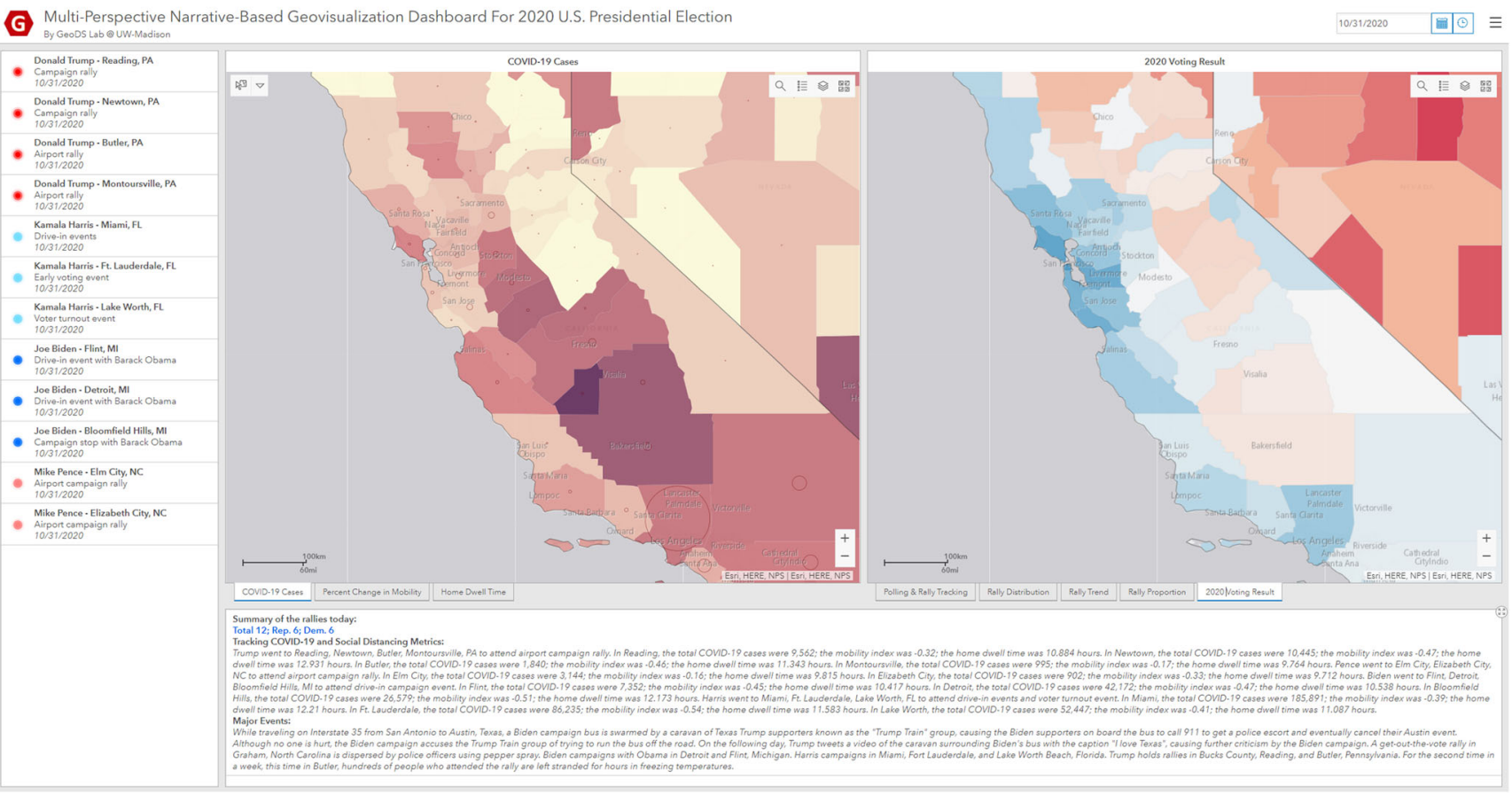

Fig. 3 The web interface of the dashboard

the relative electoral support for each candidate and reveals the states, counties, and cities that presidential candidates focused on during the campaign.

\section{Interactive Narrative}

The interactive narrative in our dashboard is powered by the functions and user interfaces designed in the methodology layer, particularly the Rally Tracking function (Fig. 6 A), the Location Synchronization function (Fig. 6 B), and a series of statistical charts (Fig. 7). As introduced in Fig. 6 A, when users select a rally item in the rally list, the web maps will all jump to the location of that rally and show the information of that rally as a popup dialog (e.g., date, city name, candidate name, plain-text description) as well as the distribution of the COVID-19 cases and social distancing metrics around the area. By clicking on the arrow-icon button on the upper-left corner of each map component, users can enter the "select" mode. In this mode, when users click on the place around the rally location (e.g., a county feature), the purple polygon indicators will flash on the same place on other associated maps. Such indicators can help users match locations across the maps. Meanwhile, the narrative panel on the bottom will show the summary of the candidates' activities, COVID-19 cases, social distancing metrics, and major events that happened on that date accordingly. Figure 6 B shows an example of the Location Synchronization in the dashboard. When users adjust (e.g., pan, zoom) the extent of any of the maps, all the other maps will synchronize their extents accordingly to match the adjusted extent. Such a function aligns the map features among all the maps simultaneously, which facilitates users to investigate multiple factors across space and over time.

\section{Insights from the Results}

Our dashboard provides spatial-temporal visualization for multiple factors (COVID-19 cases, social distancing metrics, and poll results) during the presidential campaign and takes rally and event data as clues to provide the interactive narrative for the 2020 US presidential election. Tracking and analyzing such information throughout the timeline helps us capture the campaign activity patterns of the candidates.

We start by investigating the frequency and the trend in holding rallies for each candidate and their running mate. From September 1 to November 3, 2020, as shown in Fig. 7, Donald Trump and Mike Pence held more campaign stops than Joe Biden and Kamala Harris. Trump made 79 stops in 17 states, compared to Biden's 57 stops in 13 states. One reason for such differences may be Biden's control of public gatherings for campaign events due to the COVID-19 pandemic problems. Even so, candidates from both parties significantly increased their campaign activities after the middle of October, 2020. In the last two weeks before Election Day (November 3, 2020), Donald Trump and Mike Pence held significantly more campaign rallies 
A
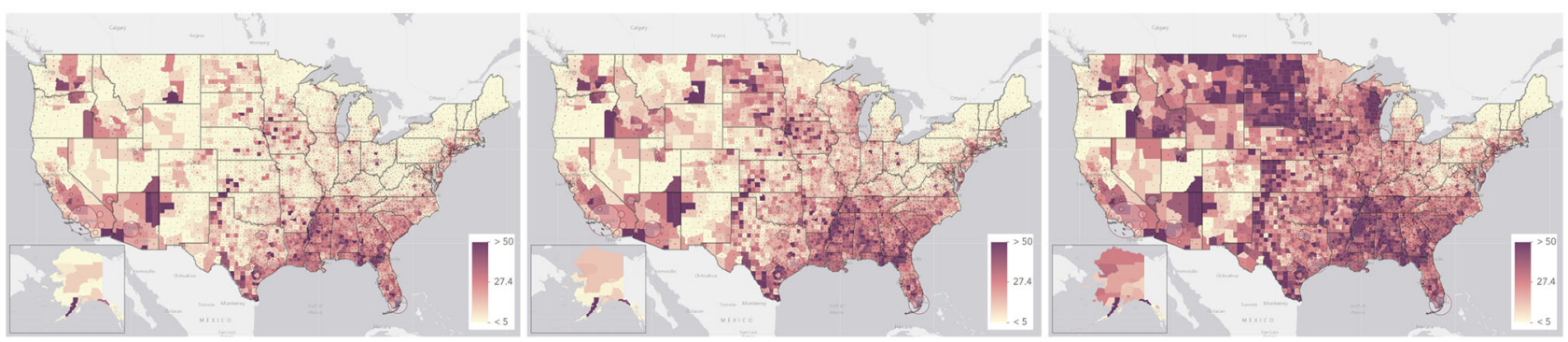

B
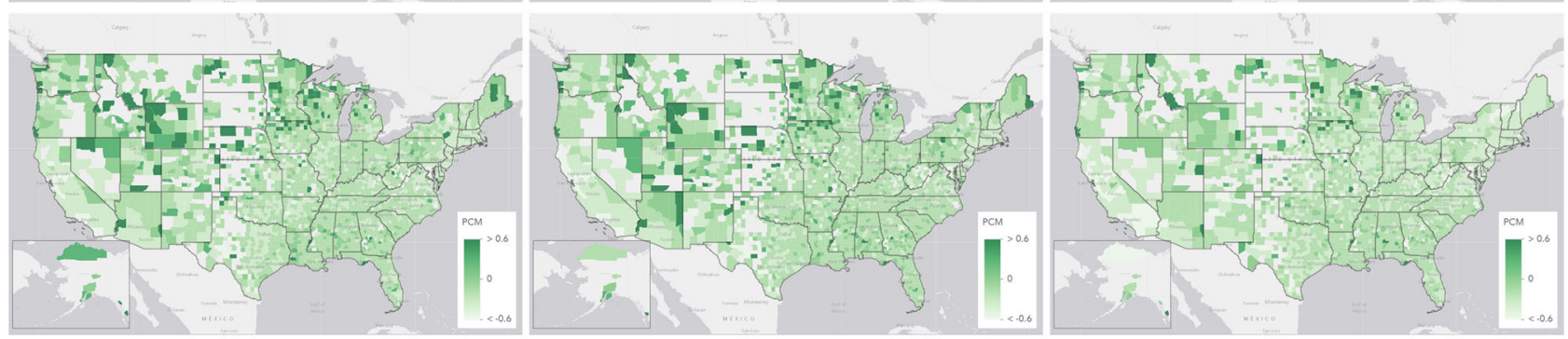

C
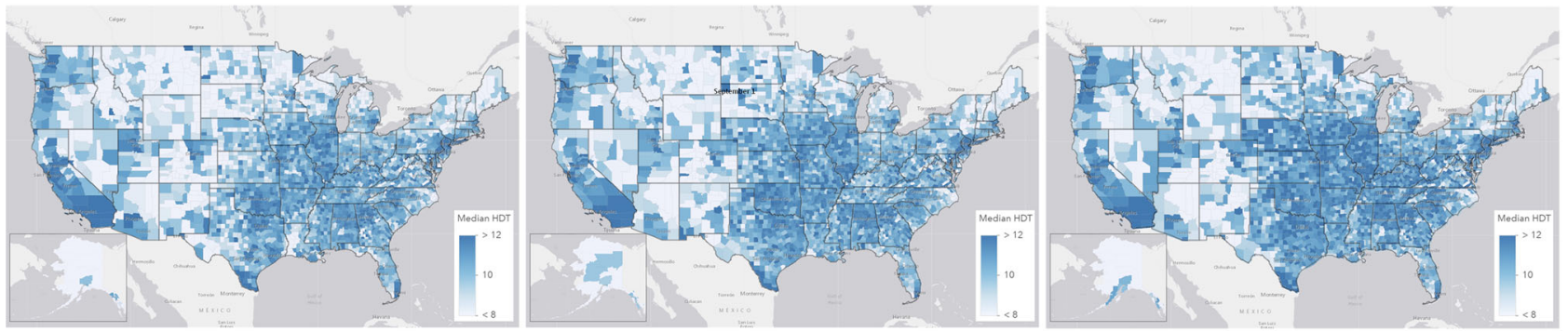

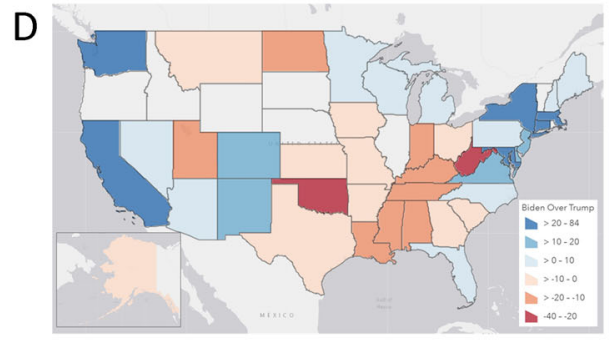

September 1, 2020

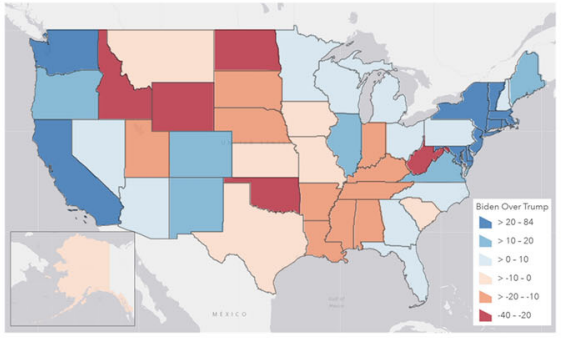

October 6, 2020

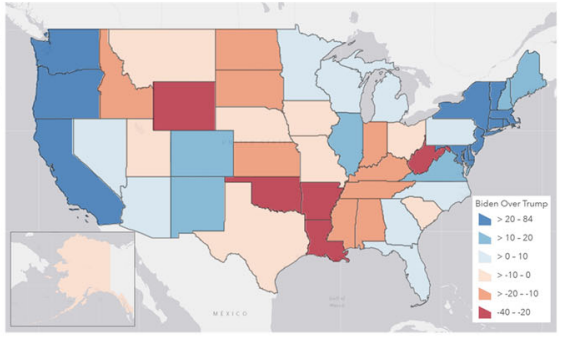

November 3, 2020
Fig. 4 Visualization of various factors across the country on September 1, 2020, October 6, 2020, and November 3, 2020 (the first Tuesday of each month). Row A: COVID-19 cases per 1000 people at the

than Joe Biden and Kamala Harris. The most intensive portion of the campaign occurred three days before Election Day, during which each candidate held approximately five events per day. Even before the polls closed, Harris and Biden were still making public appearances in Michigan and Delaware. Overall, the campaign activities slightly concentrated around mid-October towards the end of the election. As the Election Day drew nearer, the number of campaign visits increased for both parties.

From the spatial perspective, campaign rallies mostly occurred in battleground states (which can be identified with the polling estimates) such as Florida, Arizona, North Carolina, Pennsylvania, and Wisconsin. Geographically, county level. Row B: Percent change in mobility at the county level. Row C: Median home dwell time at the county level; Row D: Poll results at the state level

both candidates visited the East Coast and the South more frequently than the West. There is also a rural and urban divide of the campaign visits among the two candidates. Joe Biden and Kamala Harris rallied more often in metropolises or cities such as Detroit, Houston, and Milwaukee. In comparison, Trump's campaigns appeared to be disseminated in smaller cities like Lansing, Jupiter, and Green Bay. For example, in Wisconsin (Fig. 8), Donald Trump's team campaigned in 11 suburban counties while Joe Biden's team only visited two places and one of them was the largest city (Milwaukee) in the state. The ruralurban divide became salient for states like Wisconsin, Pennsylvania, and Georgia, where voters were split along 


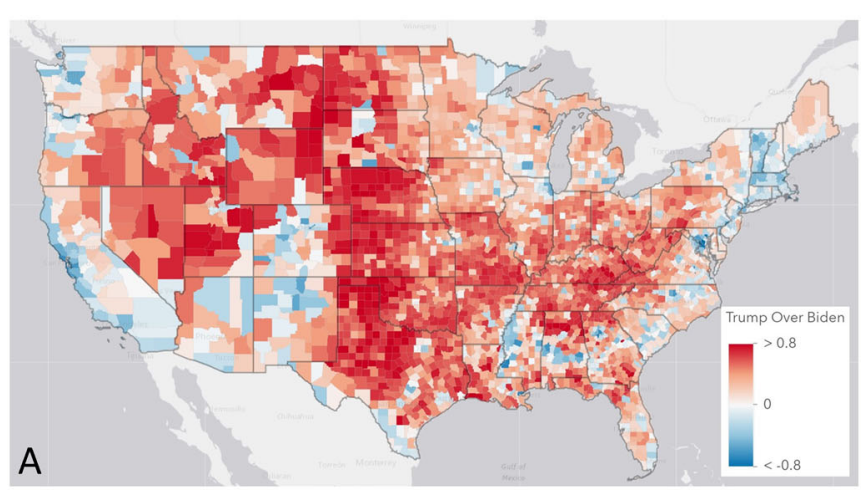

Fig. 5 Visualization of the county-level presidential election results and campaign rallies across the country from September 1 to November 3, 2020. A The difference in electoral support between Donald Trump and Joe Biden. Blue represents areas that Joe Biden won, and red represents areas that Trump won; B the rallies held by the

geographic lines. Some battlefield states, such as Florida, were less electorally divided and saw similar campaign visits in urban and suburban counties. Both candidates

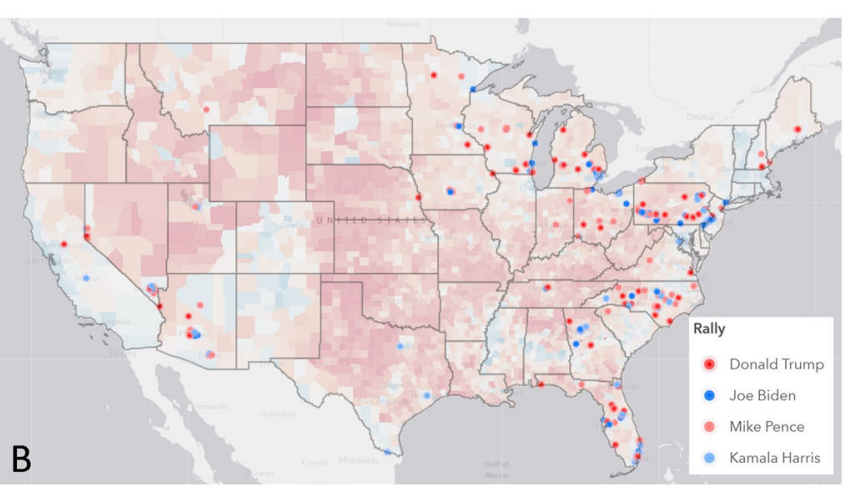

presidential candidates and their running mates. Red and light red represent the rallies held by Donald Trump and Mike Pence, respectively; blue and light blue represent the rallies held by Joe Biden and Kamala Harris, respectively

rallied in Tampa, Miami, and Jacksonville, as well as other small cities. Another notable spatial characteristic is that Biden had more virtual and drive-in campaigns,

A G Multi-Perspective Narrative-Based Geovisualization Dashboard For 2020 U.S. Presidential Election

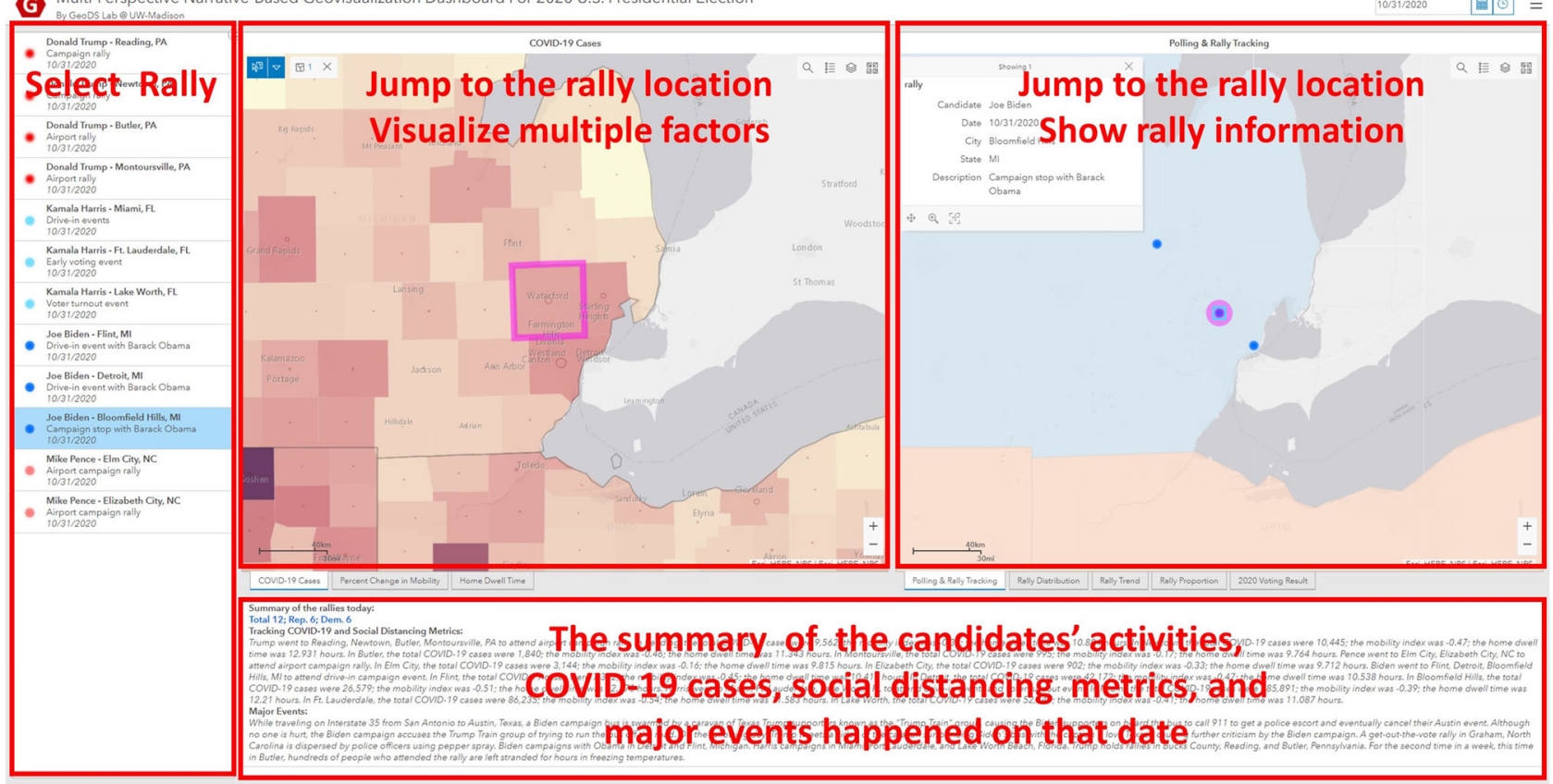

B

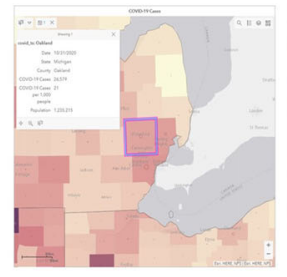

COVID-19 Cases per 1,000 People

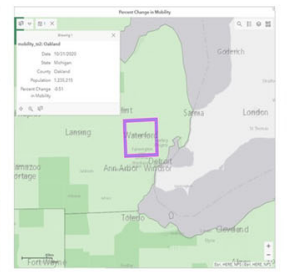

Percent Change in Mobility

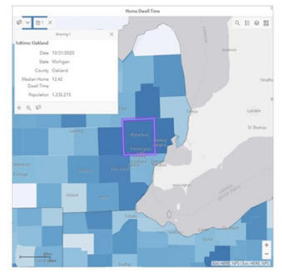

Median Home Dwell Time

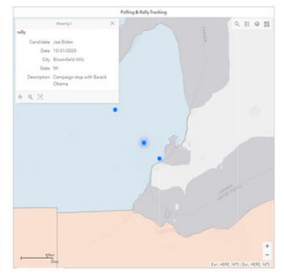

Polling \& Rally Tracking

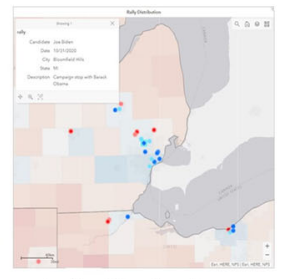

Rally Distribution

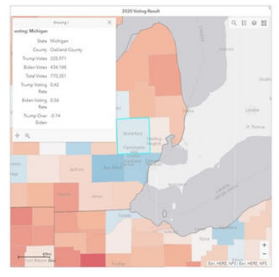

2020 Voting Result

Fig. 6 Examples of the Rally Tracking function and the Location Synchronization function. A An example of rally tracking using our dashboard; B an example of location synchronization between the web

maps of COVID-19 cases, percent change in mobility, median home dwell time, poll results, and presidential election results 
A

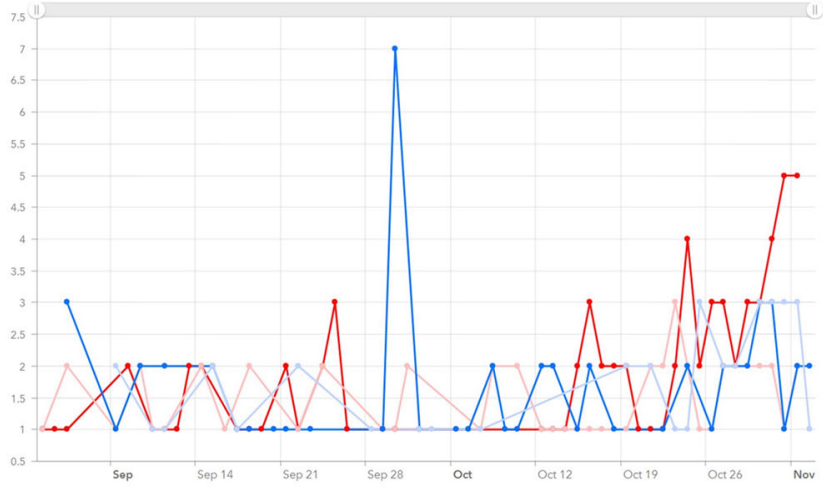

Fig. 7 The statistical charts in the dashboard. A The line chart showing the trends in the frequency of rallies held by candidates from September 1 to November 3, 2020. B The stack bar chart showing the proportions of rallies held by candidates each day from September 1 to

while Trump held more in-person rallies, likely due to the Democratic Party's advocacy for stricter policies to slow the spread of COVID-19 (Grossman et al. 2020).
B

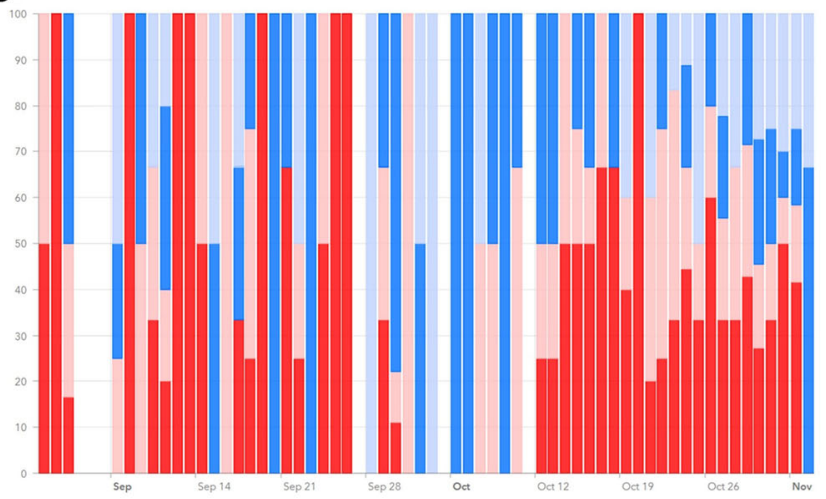

November 3, 2020. Red and light red represent the rallies held by Donald Trump and Mike Pence, respectively; blue and light blue represent the rallies held by Joe Biden and Kamala Harris, respectively

For the temporal aspect of social distancing metrics, both the percent change in mobility and the median home dwell time have regular fluctuations on a weekly basis, and the

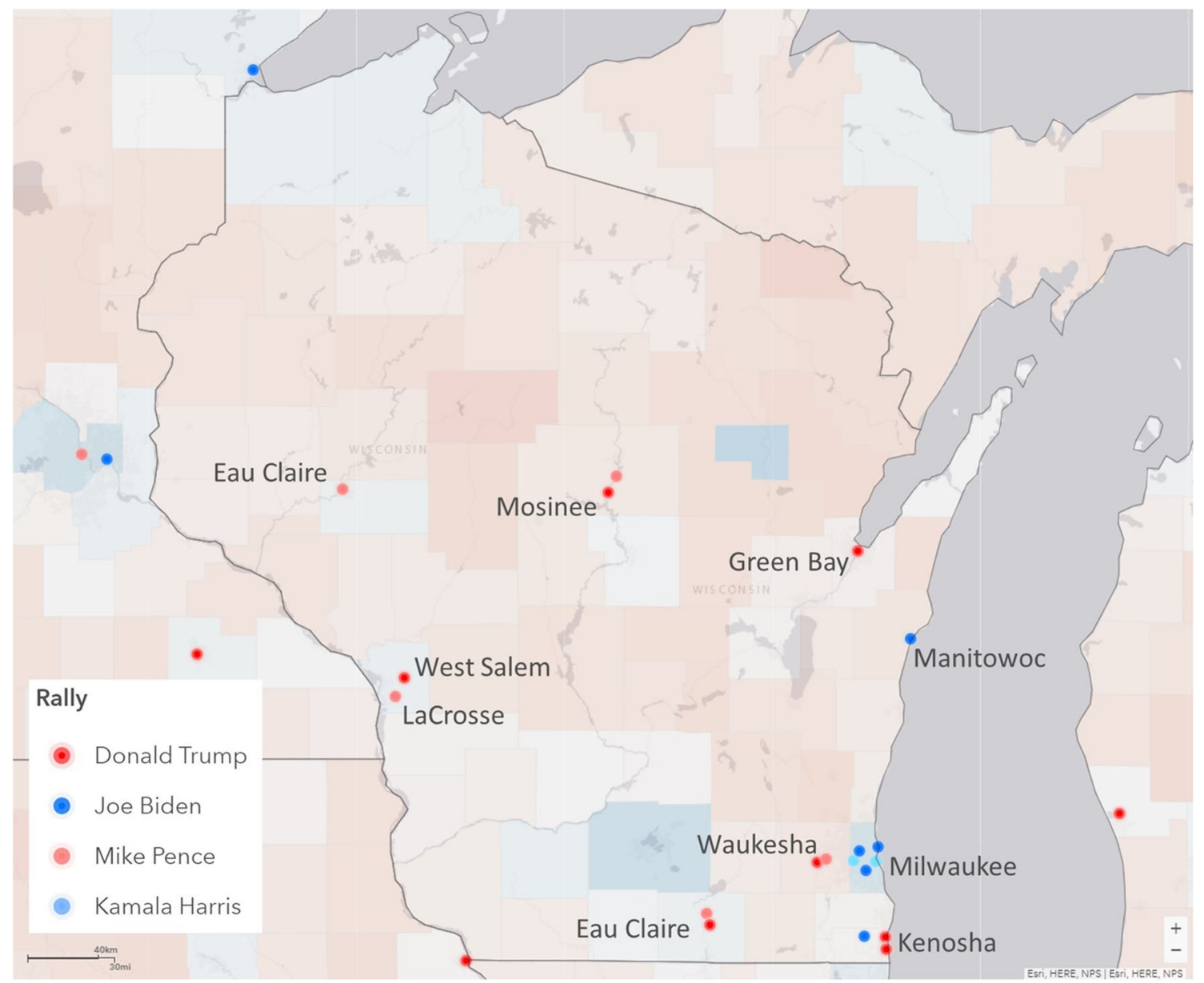

Fig. 8 The rural-urban divide of the campaign rallies by both parties in Wisconsin 
overall trends are stable. One possible reason is that after going through the early outbreak of the COVID-19 and pandemic control policies such as the stay-at-home orders and mask mandates, people start to adapt to the pandemic and gradually return to their daily lives. However, the spread of the COVID-19 still continues, and the cumulative COVID-19 cases rise from around 6 million on September 1, 2020, to over 9 million on November 3, 2020. Spatially, geographic deviations exist in the distribution of COVID-19 cases and social distancing metrics. Overall, people living on the East Coast and the West Coast have lower travel mobility and higher median home dwell time compared to residents in the Midwest and the Mountain West. Also, the number of COVID-19 cases per 1000 people has a notable growth rate in the Midwest and the South. One reason could be that the states on the East Coast and the West Coast have more effective pandemic control policies, and people better practice social distancing (Grossman et al. 2020). This may also be reflected in the polling data and electoral results, which show that residents on the East Coast and West Coast are more likely to support Biden, who is associated with stricter policies to address the pandemic. This line of inquiry demonstrates the utility of our map dashboard.

\section{User Evaluation}

We further conducted a user evaluation experiment to examine the design and efficacy of this dashboard. The participants include 12 graduate students majoring in various disciplines including Geography, Computer Science, Communication, Economics, and Biology. We asked them to interact with the dashboard and explore the content (e.g., query daily COVID-19 cases in a specific county over time, observe the spatial distribution of social distancing data and partisanship data, track the presidential campaign progress using rallies and events) through the provided web interface and functions. After their usage, we asked them to fill out our designed questionnaire to rate different aspects of the dashboard. The participants need to answer 6 questions regarding the web interface design, data, functionality, response time, exploratory capacity, and speed of comprehension. The rating results by users are shown in Table 2. Overall, the results show that the data and functions in our dashboard are sufficient, the user experience is smooth, and the interface design is suitable.

\section{Discussion}

In this section, we would like to share some of our experiences in the practical dashboard design and also discuss some limitations of our work.

\section{Practical Dashboard Design}

In this work, we utilize ArcGIS Dashboards to design and implement the dashboard since it naturally supports spatial-temporal visualization and provides many built-in interactive components that can be used for interactive narrative. In terms of the dashboard layout, the main challenge we encountered is how to integrate multiple maps into the dashboard. After several attempts, we found that stacking maps together and managing them using tabs is a good way to save space. Also, by leveraging location synchronization and dual-map-panel layout, we are able to better align the maps and associate multiple factors simultaneously (i.e., COVID-19 cases, social distancing metrics, rally locations, and poll results). In terms of interactivity, we provide both basic functions (e.g., data inquiry by clicking on a map feature) and composite functions (e.g., rally tracking) to help users better interact with the dashboard. For the composite functions, we usually need the different components to work in close cooperation so as to achieve advanced interaction such as rally tracking and location synchronization, and the key is to link the components using the FIPS code and the map extent. In terms of the symbol design, we use choropleth maps with reasonable color design to visualize COVID-19 cases per 1000 people, social distancing metrics, poll results, and the presidential election results since they can clearly exhibit the quantity and the distribution of the data, which lowers the learning cost for users. We also use proportional circles to represent the cumulative COVID-19 cases and use point
Table 2 The rating results from user evaluation

\begin{tabular}{lll}
\hline Aspect & Description & Average rating \\
\hline Interface design & Hard to use (1)-perfect (5) & 4.333 \\
Data & Limited (1)-sufficient (5) & 4.583 \\
Functionality & Limited (1)-sufficient (5) & 4.250 \\
Response time (latency) & Slow to respond (1)-smooth (5) & 4.250 \\
Exploratory capacity & Low (1)-high (5) & 4.000 \\
Speed of comprehension & Low (1)-high (5) & 4.250 \\
\hline
\end{tabular}


symbols to represent the rally locations, which are simple, clear, and accurate.

\section{Limitations}

There are some limitations in our work. First, our dashboard focuses on the rallies and events from September 1 to November 3, 2020, while many earlier events, such as the nationwide protests for George Floyd (i.e., the "Black Life Matters" movement), may also affect the presidential election and thus are worth exploring. Also, due to the limited forms of the built-in dashboard component, changing dates is currently supported by a calendarlike date picker, which is not as intuitive as a timeline slider. Second, although all the dashboard components are internally associated, the display of the narrative panel and the maps are relatively independent. Further integration of the narrative elements into the geovisualization process would be very promising. Third, due to data limitations from FiveThirtyEight, we only include the state-level poll estimates. We believe that the poll results at a finer spatial resolution (e.g., county level) would reveal the spatial heterogeneity in partisanship within a state, which may better explain the difference in the campaign strategies by the presidential candidates. Last but not least, our current dashboard is mainly about the interactive visualization, but showing the correlation analysis results on the selected location between two given data layers or showing the timelagged association between mobility indices and COVID-19 cases would be worth exploring in our future work.

\section{Conclusions}

In this work, we design and implement a narrative-based geovisualization dashboard for the 2020 US presidential election. The dashboard takes campaign rallies and major events from September 1 to November 3, 2020, as narrative clues to illustrate the process of the presidential election. Multiple-perspective factors including COVID19 infection cases, social distancing metrics, and poll results are collected, processed, and integrated into the dashboard for spatio-temporal visualization. We also design a series of interactive functions such as rally tracking and location synchronization to support the interactive narrative for the presidential campaign. Compared with traditional storytelling maps and dashboard products, our dashboard integrates the spatio-temporal visualization of multi-perspective data with interactive narrative elements, which strikes a better balance between the narrative property and the data visualization property of the dashboard. The user evaluation result shows that the data and functions in our dashboard are sufficient, the user experience is smooth, and the interface design is suitable. Our work may be used for demonstrating and associating multiple factors with partisanship and has the potential to help users explore the interaction between policies controlling COVID-19, social distancing, and partisanship across the country during the 2020 US presidential election, which brings new insights into the GIS tool development of narrative-based geovisualization for social and political events.

Funding We acknowledge the funding support provided by the National Science Foundation (Award No. BCS-2027375).

\section{Declarations}

Ethics Approval and Consent to Participate All procedures were in accordance with the ethical standards of the institutional and/or national research committee and with the 1964 Helsinki declaration and its later amendments or comparable ethical standards.

Informed Consent All human participants consent voluntarily to be participants in this study. They are informed that the research findings will be shared more broadly, for example, through publications and conferences.

Conflict of Interest The authors declare no competing interests.

Disclaimer Any opinions, findings, and conclusions or recommendations expressed in this material are those of the author(s) and do not necessarily reflect the views of the National Science Foundation.

\section{References}

Bernheim BD, Buchmann N, Freitas-Groff Z, Otero S (2020) The effects of large group meetings on the spread of COVID-19: the case of Trump rallies. Stanford Institute for Economic Policy Research (SIEPR)

Caquard S, Cartwright W (2014) Narrative cartography: from mapping stories to the narrative of maps and mapping

Caquard S, Pyne S, Igloliorte H, Mierins K, Hayes A, Taylor DF (2009) A "living" atlas for geospatial storytelling: the cybercartographic atlas of indigenous perspectives and knowledge of the great lakes region. Cartographica: Int J Geogr Inf Geovisualization 44(2):83-100

Card SK, Moran TP, Newell A (2018) The psychology of humancomputer interaction. Crc Press

Clinton J, Cohen J, Lapinski J, Trussler M (2021) Partisan pandemic: how partisanship and public health concerns affect individuals' social mobility during covid-19. Sci Adv 7(2):eabd7204

Desmet K, Wacziarg R (2020) Understanding spatial variation in covid-19 across the united states. Tech. rep. National Bureau of Economic Research

Dong E, Du H, Gardner L (2020) An interactive web-based dashboard to track covid-19 in real time. Lancet Inf Dis 20(5):533-534

Dransch D (2001) User-centered human-computer-interaction in cartographic information processing

Esri (2021) ArcGIS Dashboards. https://www.esri.com/en-us/arcgis/ products/arcgis-dashboards/overview

Few S (2006) Information dashboard design: The effective visual communication of data vol 2. O'reilly Sebastopol, CA 
Fish C (2020) Storytelling for making cartographic design decisions for climate change communication in the united states. Cartographica: Int J Geogr Inf Geovisualization 55(2):69-84

Gao S, Rao J, Kang Y, Liang Y, Kruse J (2020a) Mapping countylevel mobility pattern changes in the united states in response to covid-19. SIGSpatial Special 12(1):16-26

Gao S, Rao J, Kang Y, Liang Y, Kruse J, Dopfer D, Sethi AK, Reyes JFM, Yandell BS, Patz JA (2020b) Association of mobile phone location data indications of travel and stay-at-home mandates with covid-19 infection rates in the us. JAMA Netw Open 3(9):e2020485-e2020485

Grossman G, Kim S, Rexer JM, Thirumurthy H (2020) Political partisanship influences behavioral responses to governors' recommendations for covid-19 prevention in the united states. Proc Natl Acad Sci 117(39):24144-24153

Hanna SP, Carter PL, Potter AE, Bright CF, Alderman DA, Modlin EA, Butler DL (2019) Following the story: narrative mapping as a mobile method for tracking and interrogating spatial narratives. $\mathrm{J}$ Herit Tour 14(1):49-66

Hecht B, Schöning J, Erickson T, Priedhorsky R (2011) Geographic human-computer interaction. In: CHI'11 Extended Abstracts on Human Factors in Computing Systems, pp. 447-450

Hou X, Gao S, Li Q, Kang Y, Chen N, Chen K, Rao J, Ellenberg JS, Patz JA (2021) Intracounty modeling of covid-19 infection with human mobility: assessing spatial heterogeneity with business traffic, age, and race. Proc Natl Acad Sci 118(24):e2020524118

Iyengar S, Westwood SJ (2015) Fear and loathing across party lines: new evidence on group polarization. Am J Polit Sci 59(3):690-707

Johnson AF, Pollock W, Rauhaus B (2020) Mass casualty event scenarios and political shifts: 2020 election outcomes and the us covid-19 pandemic. Adm Theory Prax 42(2):249-264

Kerski JJ (2015) Geo-awareness, geo-enablement, geotechnologies, citizen science, and storytelling: geography on the world stage. Geogr Compass 9(1):14-26

Kitchin R, Lauriault TP, McArdle G (2015) Knowing and governing cities through urban indicators, city benchmarking and real-time dashboards. Reg Stud Reg Sci 2(1):6-28

Lazar J, Feng JH, Hochheiser H (2017) Research methods in humancomputer interaction. Morgan Kaufmann

MacEachren AM (1986) A linear view of the world: strip maps as a unique form of cartographic representation. Am Cartogr 13(1):7-26
Norris K, Gonzalez C (2020) Covid-19, health disparities and the us election. EClinicalMedicine 28

Ricker BA, Roth RE (2018) Mobile maps and responsive design. Geographic Information Science \& Technology Body of Knowledge, [CV-40]. https://doi.org/10.22224/gistbok/2018.2.5

Robinson AH (1986) The look of maps: an examination of cartographic design. Am Cartogr 13(3):280-280

Roth RE (2012) Cartographic interaction primitives: framework and synthesis. Cartogr J 49(4):376-395

Roth RE (2021) Cartographic design as visual storytelling: synthesis and review of map-based narratives, genres, and tropes. The Cartographic Journal 58(1):83-114. https://doi.org/10.1080/00087041.2019.1633103

Segel E, Heer J (2010) Narrative visualization: telling stories with data. IEEE transactions on visualization and computer graphics 16(6):1139-1148

Sides J, Tausanovitch C, Vavreck L (2020) The politics of covid19: Partisan polarization about the pandemic has increased, but support for health care reform hasn't moved at all. Harvard Data Science Review

Sun F, Matthews SA, Yang TC, Hu MH (2020) A spatial analysis of the covid-19 period prevalence in us counties through june 28, 2020: where geography matters? Ann Epidemiol 52:54-59

Tilley I, Pettit C (2020) A dashboard for the unexpected: open data for real-time disaster response, Springer

Warren MS, Skillman SW (2020) Mobility changes in response to covid-19. arXiv:200314228

Wood CH, Keller CP (1996) Cartographic design: theoretical and practical perspectives. Wiley, Chichester

Wood D (1987) Pleasure in the idea/the atlas as narrative form. Cartographica: Int J Geogr Inf Geovisualization 24(1):24-46

Ye X, Du J, Gong X, Na S, Li W, Kudva S (2021) Geospatial and semantic mapping platform for massive covid-19 scientific publication search. J Geovisualization Spatial Anal 5(1):112

Zuo C, Ding L, Meng L (2020) A feasibility study of map-based dashboard for spatiotemporal knowledge acquisition and analysis. ISPRS Int J Geo-Inform 9(11):636

Publisher's Note Springer Nature remains neutral with regard to jurisdictional claims in published maps and institutional affiliations. 